\title{
THE COHOMOLOGY OF CERTAIN FUNCTION SPACES
}

\author{
MARTIN BENDERSKY AND SAM GITLER
}

\begin{abstract}
We study a spectral sequence converging to the cohomology of the configuration space of $n$ ordered points in a manifold. A chain complex is constructed with homology equal to the $E_{2}$ term. If the field is the rationals and the manifold is formal then the spectral sequence is shown to collapse. The results are applied to compute the Anderson spectral sequence converging to the cohomology of a function space.
\end{abstract}

\section{INTRODUCTION}

From the point of view of homotopy theory, a very natural problem is to describe the topology of function spaces. In 1956, R. Thom [13] wrote a seminal paper. In 1972, D. Anderson [1], using the notion of a cosimplicial space introduced by Bousfield and Kan [4], obtained a spectral sequence for the homology of function spaces. In 1969-1970, Gelfand and Fuks [6] wrote a series of papers on the continuous cohomology of the Lie algebra of vector fields on a manifold $M$. They introduced a pair of spectral sequences for such a study. Based on the results of Gelfand-Fuks, Bott and Fuks conjectured that the continuous cohomology of the Lie algebra of vector fields was the usual cohomology of a certain function space, namely, that of the space of sections of a certain associated bundle to the tangent bundle of $M$.

Haëfliger [7] and Bott and Segal [3] in 1978, using entirely different methods, proved the Bott-Fuks conjecture. The method followed by Bott and Segal was to utilize the ideas of Anderson.

The cohomology of "configuration spaces" appears in the computation of the $E_{1}$-term of the above spectral sequences. In 1978, F. Cohen and L. Taylor [5] were able to compute the cohomology of the configuration spaces for a certain class of manifolds, and applied these results to describe the Gelfand-Fuks cohomology of these manifolds. Also, there has been interest in the configuration spaces as they appear as approximations to certain function spaces (see [2, 9]). In this paper we study the configuration spaces and the function spaces as outlined by Bott-Segal. In both instances the cohomology can be obtained from a

Received by the editors October 3, 1987 and, in revised form, July 1, 1989.

1980 Mathematics Subject Classification (1985 Revision). Primary 54C35.

Key words and phrases. Configuration space, simplicial space, formal space.

This paper was prepared while both authors were visiting the Institute for Advanced Study.

The second author was partially supported by NSF Grant MCS-8108814 (A04) and CONACYT Mexico. 
double complex. A double complex comes with its two natural filtrations and thus produces two spectral sequences.

If we take the rationals as the coefficient groups, we may use the Sullivan cochains [12] which are commutative, and these double complexes become functors on the cochain algebras which preserve weak equivalences. Recall that a space $X$ is formal if its Sullivan rational cochain algebra is weakly equivalent to its rational cohomology algebra.

For formal spaces, collapsing results on the spectral sequences emerge and thus also some computational theorems (see $\S \S 3$ and 7):

Also, the two spectral sequences of Gelfand-Fuks appear when we describe the Bott-Segal spectral sequence for the space of sections in $\S 7$.

This paper grew thus from our attempts to understand the work of GelfandFuks-Bott-Segal on the one hand, and that of Segal, Cohen, Taylor, McDuff [9] and Bödigheimer [2] on the other. We want to thank E. Antoniano, W. Browder, E. H. Brown, G. Carlson, F. R. Cohen, M. Hopkins, and D. M. Kan for many helpful and illuminating discussions while trying to understand the results presented here.

\section{The SPECTRAL SEQUeNCE OF A SiMPlicial SPACE}

Recall from [4] the notion of a simplicial space. Let $\Delta^{p}$ be the standard $p$-simplex; $\Delta^{p} \subset R^{p+1}$ consists of points $t=\left(t_{0}, \ldots, t_{p}\right)$ with $0 \leq t_{i} \leq 1$ and $\sum t_{i}=1, \eta_{i}: \Delta^{p-1} \rightarrow \Delta^{p}, i=0, \ldots, p$, given by

$$
\eta_{i}\left(t_{0}, \ldots, t_{p-1}\right)=\left(t_{0}, \ldots, t_{i-1}, 0, t_{i}, \ldots, t_{p-1}\right),
$$

and $\varepsilon_{i}: \Delta^{p} \rightarrow \Delta^{p-1}, i=0, \ldots, p-1$, given by

$$
\varepsilon_{i}\left(t_{0}, \ldots, t_{p}\right)=\left(t_{0}, \ldots, t_{i-1}, t_{i}+t_{i+1}, \ldots, t_{p}\right) .
$$

A simplicial space $\mathbf{X}=\left\{X_{p}\right\}_{p=0}^{\infty}$ is a family of spaces $X_{p}$ and continuous maps

$$
X_{p} \stackrel{\partial_{i}}{\rightarrow} X_{p-1}, \quad i=0,1, \ldots, p, \quad X_{p-1} \stackrel{s_{j}}{\rightarrow} X_{p}, \quad j=0, \ldots, p-1,
$$

satisfying the simplicial identities; namely,

$$
\begin{aligned}
& \partial_{i} \partial_{j}=\partial_{j-1} \partial_{i} \quad \text { if } i<j, \\
& s_{i} s_{j}=s_{j+1} s_{i} \quad \text { if } i \leq j, \\
& \partial_{i} s_{j}= \begin{cases}s_{j-1} \partial_{i} & \text { if } i<j, \\
\text { id } & \text { if } i=j, j+1, \\
s_{j} \partial_{i-1} & \text { if } i>j+1 .\end{cases}
\end{aligned}
$$

$\mathbf{X}$ has a geometric realization $|\mathbf{X}|$ obtained as the quotient space of $\amalg \Delta^{p} \times X_{p}$ by the relations

$$
\begin{array}{ll}
\left(t, \partial_{i} x\right) \sim\left(\eta_{i} t, x\right), & x \in X_{p}, t \in \Delta^{p-1}, \\
\left(t, s_{i} x\right) \sim\left(\varepsilon_{i} t, x\right), & x \in X_{p}, t \in \Delta^{p+1} .
\end{array}
$$


Example 1.1. Let $Y$ be a topological space and $\left\{U_{i}\right\}_{i \in A}$ an open covering of $Y$. If $I=\left(i_{1}, \ldots, i_{k}\right)$ is a sequence of integers $i_{1} \leq i_{2} \leq \cdots \leq i_{k}$ belonging to $A$, we denote by $l(I)=k$ the length of $I$ and by $U_{I}$ the intersection $U_{i_{1}} \cap \cdots \cap U_{i_{k}}$, when it is not empty. Let $V_{0}=\amalg U_{i}, \ldots, V_{p}=\bigsqcup_{l(I)=p+1} U_{I}, \ldots$. Then we may define the face and degeneracy maps using the inclusions and repetitions; thus, $\left\{V_{p}\right\}=\mathbf{V}$ is a simplicial space. In this case $|\mathbf{V}|$ is weakly equivalent to $Y$.

It is helpful to display the simplicial data $V_{i}, \partial_{i}$ and $s_{i}$ in a diagram:

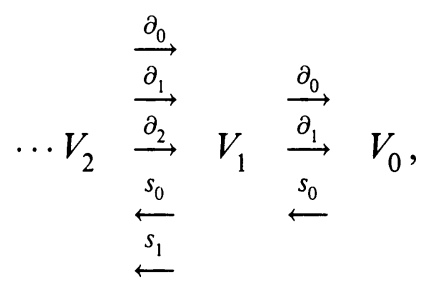

where, for example, the face and degeneracy maps connecting $V_{1}$ and $V_{0}$ are defined by

$$
\partial_{0}:\left(U_{i_{1}} \cap U_{i_{2}}\right) \rightarrow U_{i_{2}}, \quad \partial_{i}:\left(U_{i_{1}} \cap U_{i_{2}}\right) \rightarrow U_{i_{1}}
$$

(i.e., the 0 th or 1 st term respectively is deleted from the intersection), and $s_{0}:\left(U_{i}\right) \rightarrow U_{i} \cap U_{i}$.

The situation of two open sets covering $Y$ is related to the Meyer-Vietoris sequence. In this case the geometric realization is (essentially)

$$
\left\{U_{1} \cup U_{2}\right\} \cup\left(I \times U_{1} \cap U_{2}\right) / \sim,
$$

where $\sim$ glues $U_{1}$ and $U_{2}$ to the top and bottom of $\left(I \times U_{1} \cap U_{2}\right)$. This is clearly the homotopy type of $Y$.

We may also consider a simplicial complex $K$ with $K=\bigcup K_{i}$, where the $K_{i}$ are the subcomplexes, and proceed as above to define a simplicial space $\left\{V_{p}\right\}=\mathbf{V}$ and again $|\mathbf{V}|$ is of the same homotopy type as $K$.

A chain functor is a functor from the category of topological spaces and continuous maps to the category of chain complexes. We assume there is an Eilenberg-Zilber chain map,

$$
C_{*}(X) \otimes C_{*}(Y) \rightarrow C_{*}(X \times Y),
$$

which we assume is functorial in each variable. (The differential in $C_{*}(X) \otimes$ $C_{*}(Y)$ is induced from the differentials in $C_{*}$ by $\partial(x \otimes y)=\partial x \otimes y+(-1)^{|x|} x \otimes$ $\partial y$.) An example of such a functor is the simplicial chain functor.

Given the simplicial space $\mathbf{X}$ and a chain functor $C_{*}$, we may form $\left\{C_{*}\left(X_{p}\right)\right\}$ which is a double complex with the following differentials:

$$
d_{1}=\partial: C_{q}\left(X_{p}\right) \rightarrow C_{q-1}\left(X_{p}\right), \quad d_{2}=\bar{\partial}: C_{q}\left(X_{p}\right) \rightarrow C_{q}\left(X_{p-1}\right),
$$

where $\bar{\partial}=\sum(-1)^{i}\left(\partial_{i}\right)_{\#}$. Since $C_{*}$ is functorial, $\partial$ commutes with $\bar{\partial}$. 
Letting $C_{n}=\sum_{p+q=n} C_{q}\left(X_{p}\right)$ and $d=\bar{\partial}+(-1)^{p} \partial$, we define a chain complex $(C, d)$, which we will denote by $\left|C_{*}(\mathbf{X})\right|$.

We have natural maps $\Delta^{p} \times X_{p} \stackrel{\theta_{p}^{*}}{\rightarrow}|X|$ and a commutative diagram:

Let $\bar{\theta}_{p}$ denote the composite

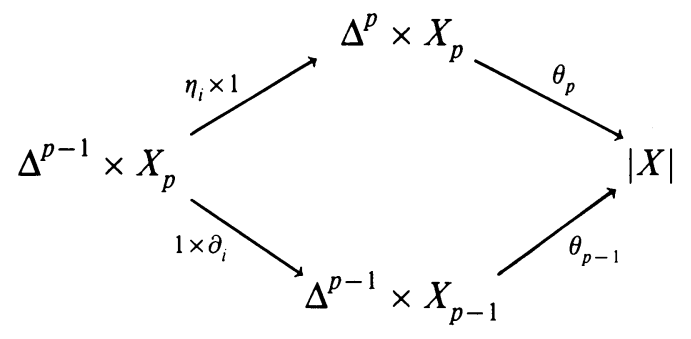

$$
C_{*}\left(\Delta^{p}\right) \otimes C_{*}\left(X_{p}\right) \stackrel{e}{\rightarrow} C_{*}\left(\Delta^{p} \times X_{p}\right) \rightarrow C_{*}(|X|),
$$

where $e$ is the Eilenberg-Zilber map. Then $\bar{\theta}_{p}$ is a chain map. Let

$$
\phi_{p}: C_{q}\left(X_{p}\right) \rightarrow C_{p}\left(\Delta^{p}\right) \otimes C_{q}\left(X_{p}\right)
$$

be defined by $\phi_{p}(x)=i_{p} \otimes x$, where $i_{p}$ is a canonical class. Define

$$
\rho: \bigoplus_{p+q=n} C_{q}\left(X_{p}\right) \rightarrow C_{n}(|X|)
$$

for all $n$ by $\rho(x)=\bar{\theta}_{p} \phi_{p}(x)$ if $x \in C_{q}\left(X_{p}\right)$. Consider $x \in C_{q}\left(X_{p}\right)$. Then

$$
\begin{aligned}
\rho(d x) & =\rho(\bar{\partial} x)+(-1)^{p} \rho(\partial x)=\bar{\theta}_{p-1}\left(i_{p-1} \otimes \bar{\partial} x\right)+(-1)^{p} \bar{\theta}_{p}\left(i_{p} \otimes \partial x\right) \\
& =\bar{\theta}_{p-1}\left(i_{p-1} \otimes \sum(-1)^{i}\left(\partial_{i}\right)_{\#} x\right)+(-1)^{p} \bar{\theta}_{p}\left(i_{p} \otimes \partial x\right),
\end{aligned}
$$

and, by the above diagram,

$$
\bar{\theta}_{p-1}\left(i_{p-1} \otimes\left(\partial_{i}\right)_{\#} x\right)=\bar{\theta}_{p}\left(\eta_{i} i_{p-1} \otimes x\right) .
$$

Thus, $\rho(d x)=\bar{\theta}_{p}\left(\partial i_{p} \otimes x\right)+(-1)^{p} \bar{\theta}_{p}\left(i_{p} \otimes \partial x\right)=\bar{\theta}_{p}\left(\partial\left(i_{p} \otimes x\right)\right)=\partial \bar{\theta}_{p}\left(i_{p} \otimes x\right)=$ $\partial \rho(x)$, i.e., $\rho$ is a chain mapping.

In a similar fashion, we can pass to cochains and define a cochain mapping

$$
\rho^{\#}: C^{n}(|X|) \rightarrow \bigoplus_{p+q=n} C^{q}\left(X_{p}\right)
$$

We may pass to normalized cochains; let

$$
\bar{C}^{*}\left(X_{p}\right)=C^{*}\left(X_{p}\right) /\left(s_{0}^{\#} C^{*}\left(X_{p+1}\right)+\cdots+s_{p}^{\#} C^{*}\left(X_{p+1}\right)\right) .
$$

Proposition 1.2. Let $\mathbf{X}$ be a simplicial space. Then the mapping $\rho^{\#}$ induces an isomorphism in cohomology

$$
\rho^{\#}: H^{n}(|X|) \rightarrow H^{n}\left(\left|\bar{C}^{*}(\mathbf{X})\right|\right) .
$$

Proof. We have natural filtrations

$$
F_{q}(\mathbf{X})=\bigcup\left\{X_{n}\right\}_{n \leq q} \cup \bigcup\left\{D(X)_{n}\right\}_{q<n},
$$


where $D(X)_{n}$ is the subspace of $X_{n}$ generated by the image of the degeneracies coming from $X_{m}, m \leq q$. Also, $|X|$ has a filtration coming from $\amalg_{n \leq q} \Delta^{n} \times$ $X_{n}$. The mapping $\rho^{\#}$ preserves the filtrations and induces a map of spectral sequences, which is easily seen to be an isomorphism in $E_{1}$.

Suppose we have $|X| \subset Y$. Then since $X_{0} \subset|X|$, we may form the cochain complex

$$
C^{*}\left(X_{-1}\right) \stackrel{\varepsilon}{\rightarrow} C^{*}\left(X_{0}\right) \rightarrow \cdots \stackrel{\bar{\delta}}{\rightarrow} C^{*}\left(X_{n}\right) \rightarrow \cdots,
$$

where $X_{-1}=Y$ and $\varepsilon$ is the induced map in cochains of the inclusion $X_{0} \subset$ $|X| \subset Y$. Then (i) is a double complex and we denote by $\left|C^{*}(Y, X)\right|$ the associated total complex. Note that now

$$
\left|C^{*}(Y, X)\right|^{n}=\bigoplus_{p+q=n-1} C^{q}\left(X_{p}\right) .
$$

Proposition 1.3. If $|X| \subset Y$, then $\left|C^{*}(Y, X)\right|^{n}$ is cochain equivalent to $C^{*}(Y,|X|)$.

Having the double complexes $C^{*}(\mathbf{X})$ and $C^{*}(Y, \mathbf{X})$ we may produce two spectral sequences. We will be interested mainly in the one that has $E_{1}^{p} \simeq$ $H^{*}\left(X_{p}\right)$ in the absolute case. In the relative case, $E_{1}^{p} \simeq H^{*}\left(X_{p-1}\right)$.

\section{The fat diagonal}

Let $K$ be a simplicial set and $K^{n}$ the $n$-fold cartesian product of $K$, $\left(K^{n}\right)_{q}=K_{q} \times \cdots \times K_{q}$ with face and degeneracies $\partial_{i} \times \cdots \times \partial_{i}$ and $s_{j} \times \cdots \times s_{j}$.

Let $D^{n} K$ be the subsimplicial set of $K^{n}$,

$$
\left(D^{n} K\right)_{q}=\left\{\sigma_{1}, \ldots, \sigma_{q} \mid \sigma_{i}=\sigma_{j}, \text { some pair } i \neq j\right\} .
$$

Then $D^{n} K=\bigcup D_{i j}^{n} K$, where $D_{i j}^{n} K=\left\{\left(\sigma_{1}, \ldots, \sigma_{q}\right) \mid \sigma_{i}=\sigma_{j}\right\}$.

We may apply the ideas of $\S 1$ to construct a double complex for $C^{*}\left(D^{n} K\right)$ and $C^{*}\left(K^{n}, D^{n} K\right) . D^{n} K$ is called the fat diagonal. Given a topological space $X$, let $D^{n} X=\left\{\left(x_{1}, \ldots, x_{n}\right) \mid x_{i} \in X, x_{i}=x_{j}\right\}$, some pair $i \neq j$.

Proposition 2.1. $\left|D^{n} K\right|$ and $D^{n}|K|$ are of the same homotopy type.

We now want to describe the spectral sequence associated to the fat diagonal. Assume $K$ is a simplicial complex, triangulared so that $K^{n}$ has $D^{n} K$ and $D_{i j}^{n} K$ as subcomplexes. Assume that $n$ is fixed, so that we will write $D K$ and $D_{i j} K$ instead of $D^{n}$.

Example 1.1 applies to $D_{k}$ with covering $\left\{D_{i j}\right\}$. The set of $(k-1)$-simplices, $V_{k-1}$, is the disjoint union of spaces $D_{I}=D_{\left(i_{1}, j_{2}\right)} \cap \cdots \cap D_{\left(i_{k}, j_{k}\right)}$ where $I=$ $\left\{\left(i_{1}, j_{2}\right), \ldots,\left(i_{k}, j_{k}\right)\right\}$ is a sequence of 2-tuples of integers $(a, b)$ with $1 \leq$ $a<b \leq n$. It is clear that $D_{I}$ is homeomorphic to $K^{r(I)}$ for some function $r$ of $I$. To describe $r(I)$ we let $G(I)$ denote a graph with vertices $\{1,2, \ldots, n\}$ 
and edges $\left(i_{1}, j_{1}\right), \ldots,\left(i_{k}, j_{k}\right) . G(I)$ breaks into $C_{1}, \ldots, C_{t}$ connected components, and

$$
D_{I}=\left\{\left(x_{1}, \ldots, x_{n}\right) \mid x_{i}=x_{j} \text { iff } i, j \in C_{m} \text { for some } m\right\} .
$$

Hence $r(I)=t=$ the number of connected components of $G(I)$. For example if $n=7$, and $I=\{(1,6),(2,4),(2,5)\}$ then:

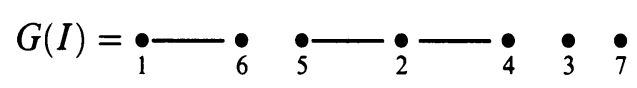

$D_{I}=\left\{\left(x_{1}, \ldots, x_{7}\right) \mid x_{i}=x_{j}\right.$ iff $i$ and $j$ are in the same component of $\left.G(I)\right\}$ and $D_{I}$ is homeomorphic to $K^{4}$. Let $|I|=$ length of $I=k$. We thus have

Proposition 2.2. The spectral sequence for $\left(K^{n}, D^{n} K\right)$ has

$$
E_{1}^{q}=\bigoplus_{|I|=q} H^{*}\left(K^{r(I)}\right) .
$$

We now want to describe the $E_{2}$-term of the spectral sequence. Let $R$ be some ground ring and let $\Lambda\left(\begin{array}{l}n \\ 2\end{array}\right)$ be an exterior algebra over $R$ on generators $\lambda_{i j}, 1 \leq i<j \leq n$, graded by $\left|\lambda_{i j}\right|=1$. Let $\sigma=\sum \lambda_{i j}$. Then $\sigma^{2}=0$, so if we define $d: \Lambda\left(\begin{array}{l}n \\ 2\end{array}\right) \rightarrow \Lambda\left(\begin{array}{c}n \\ 2\end{array}\right)$ by $d x=\sigma x, d$ is a differential and $\left(\Lambda\left(\begin{array}{l}n \\ 2\end{array}\right), d\right)$ is acyclic. To see this notice that $\Lambda$ is isomorphic to the exterior algebra on generators, $\left\{\lambda_{i j}, \sigma \mid(i, j) \neq(1,2)\right\}$. So every element annihilated by $\sigma$ is divisible by $\sigma$.

Now let $A$ be a commutative differential graded augmented algebra over $R$ and $A^{(n)}$ the $n$-fold tensor product of $A$. We have embeddings $e_{i}: A \rightarrow A^{(n)}$ given by $e_{i}(a)=1 \otimes \cdots \otimes 1 \otimes a \otimes 1 \otimes \cdots \otimes 1$, where $a$ appears in the $i$ th position, and projections $p_{i}: A^{(n)} \rightarrow A, p_{i}\left(a_{1} \otimes \cdots \otimes a_{n}\right)=\varepsilon\left(a_{1}\right) \otimes \cdots \otimes a_{i} \otimes \varepsilon\left(a_{i+1}\right) \otimes$ $\cdots \otimes \varepsilon\left(a_{n}\right)$, where $\varepsilon: A \rightarrow R$ is the augmentation.

Let $L_{n}$ be the ideal in $\Lambda\left(\begin{array}{l}n \\ 2\end{array}\right) \otimes A^{(n)}$ generated by elements of the form $\lambda_{i j} \otimes$ $\left(e_{i}-e_{j}\right)(a)$, and let $\Lambda(n, A)$ be the quotient algebra. The algebra $\Lambda\left(\begin{array}{l}n \\ 2\end{array}\right) \otimes A^{(n)}$ has two differentials, one coming from $d$ in $\Lambda\left(\begin{array}{l}n \\ 2\end{array}\right)$ and another from $A$. These induce two differentials $d_{1}, d_{2}$ in $\Lambda(n, A)$ with $d_{1} d_{2}=d_{2} d_{1}$, so we can define a total complex $\Lambda(n, A)$ with $d=d_{1}+d_{2}$. We will consider $H^{*}(\Lambda(n, A))$ to be the cohomology of this double complex.

Proposition 2.3. If $R$ is a field and $\phi: A \rightarrow B$ is a weak equivalence, then $\Lambda(\phi): \Lambda(n, A) \rightarrow \Lambda(n, B)$ is a weak equivalence.

Proof. Filter $\Lambda(n, A)$ and $\Lambda(n, B)$ so that $E_{1}=\Lambda\left(n, H^{*}(A)\right)$ and $E_{1}=$ $\Lambda\left(n, H^{*}(B)\right)$, and now $H^{*}(A) \simeq H^{*}(B)$ as algebras.

We assume that $R$ is a field, so that $H^{*}(X \times Y) \simeq H^{*}(X) \otimes H^{*}(Y)$. For any sequence $I$, define

$$
\phi_{I}: H^{*}(K)^{r(I)} \rightarrow H^{*}(K)^{(n)}
$$


as follows: Given the graph $G(I)$, suppose $C_{1}, \ldots, C_{r(I)}$ are its components and $v_{1}, \ldots, v_{r(I)}$ its leading vertices, i.e., $v_{i}$ is the smallest vertex in $C_{i}$. Then

$$
\phi_{I}\left(x_{1} \otimes \cdots \otimes x_{r(I)}\right)=y_{1} \otimes \cdots \otimes y_{n},
$$

where

$$
y_{i}= \begin{cases}1 & \text { if } i \text { is not a leading vertex } \\ x_{j} & \text { if } i \text { is the } j \text { th leading vertex. }\end{cases}
$$

Example 2.4. Take $n=7$ and $I=((1,6),(2,4),(2,5))$. Then $G(I)$ has components $C_{1}=(1,6), C_{2}=(2,4,5), C_{3}=(3), C_{4}=(7)$, and

$$
\phi_{I}\left(x_{1} \otimes x_{2} \otimes x_{3} \otimes x_{4}\right)=x_{1} \otimes x_{2} \otimes x_{3} \otimes 1 \otimes 1 \otimes 1 \otimes x_{4} .
$$

For a sequence $I=\left\{\left(i_{1}, j_{1}\right), \ldots,\left(i_{k}, j_{k}\right)\right\}$ we denote by $\lambda_{I} \otimes_{T} H^{*}(K)^{(n)}$ the image of $\lambda_{i_{1} j_{1}} \lambda_{i_{2} j_{2}} \cdots \lambda_{i_{k} j_{k}} \otimes H^{*}(K)^{(n)}$ in $\Lambda\left(H^{*}(K), n\right)$. We have a natural projection

$$
\pi_{I}: H^{*}(K)^{(n)} \rightarrow \lambda_{I} \otimes_{T} H^{*}(K)^{(n)} .
$$

Proposition 2.5. The composite mapping $\hat{\phi}_{I}$,

$$
H^{*}(K)^{r(I)} \stackrel{\phi_{I}}{\longrightarrow} H^{*}(K)^{(n)} \stackrel{\pi_{1}}{\longrightarrow} \lambda_{I} \otimes_{T} H^{*}(X)^{(n)},
$$

is an isomorphism.

Proof. It is easy to see that $\phi_{I}$ is an injection and that $\pi_{I} \circ \phi_{I}$ is also. Now induction shows that both vector spaces have the same rank.

Proposition 2.6. We have the following commutative diagram:

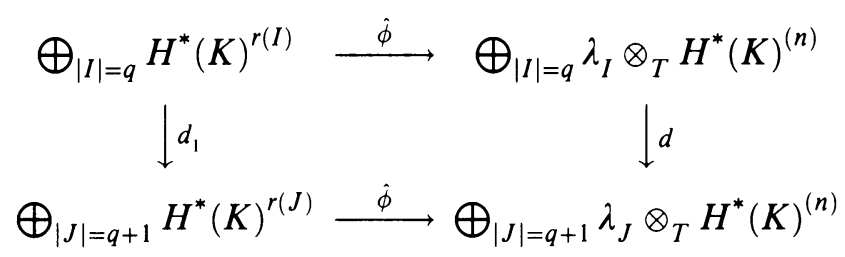

Proof. This follows by inspection.

Theorem 2.7. For any field of coefficients, there exists a spectral sequence converging to $H^{*}\left(K^{n}, D^{n} K\right)$ having $E_{2}=H^{*}\left(\Lambda\left(n, H^{*}(K)\right)\right.$.

If $K$ is the simplicial complex associated to a closed compact connected $k$-dimensional manifold, then by Lefschetz duality

$$
H^{*}\left(K^{n}, D^{n} K\right) \simeq H^{n k-*}\left(C_{n}(K)\right)
$$

as vector spaces, where $C_{n}(K)$ is the configuration space of $n$ ordered distinct points in $K$ [5]. Thus the spectral sequence of (2.7) converges additively to the cohomology of the configuration space of such a manifold.

The higher differentials in this spectral sequence in general are nontrivial, and we conjecture that they are determined by higher-order Massey products. 
Support for this conjecture is the collapsing of the spectral sequence over $Q$ in the case of a formal space (see Theorem 3.2).

Let $\Sigma_{n}$ denote the symmetric group on $n$ letters. Then $\Sigma_{n}$ acts on $\Lambda(n, A)$ as follows: For $1 \leq i<j \leq n$ let $\lambda_{j i}=\lambda_{i j}$. For $I=\left(\left(i_{1}, j_{1}\right), \ldots,\left(i_{k}, j_{k}\right)\right)$ and $\sigma \in \Sigma_{n}$, let $\sigma I$ denote $\left(\left(\sigma_{i_{1}}, \sigma_{j_{1}}\right), \ldots,\left(\sigma\left(i_{k}\right), \sigma\left(j_{k}\right)\right)\right)$. Then

$$
\sigma\left(\lambda_{I} \otimes\left(x_{1} \otimes \cdots \otimes x_{n}\right)\right)=\lambda_{\sigma I} \otimes\left(x_{\sigma(1)} \otimes \cdots \otimes x_{\sigma(n)}\right) .
$$

Proposition 2.8. The spectral sequence of (2.7) is a spectral sequence of $\Sigma_{n}$ modules.

\section{RATIONAL COHOMOLOGY OF THE FAT DIAGONAL}

It is the object of this section to prove

Theorem 3.1. If $\Omega^{*}(K)$ are the Sullivan cochains on $K$, then

$$
H^{*}\left(K^{n}, D^{n} K ; Q\right) \simeq H^{*}\left(\Lambda\left(n, \Omega^{*} K\right) ; Q\right) .
$$

Then because $\Lambda$ preserves weak equivalences, this theorem immediately produces

Theorem 3.2. If $K$ is a rationally formal space, then

$$
H^{*}\left(K^{n}, D^{n} K ; Q\right) \simeq H^{*}\left(\Lambda\left(n, H^{*}(K ; Q)\right)\right.
$$

as vector spaces.

Proof of Theorem 3.1. It suffices to see that the following diagram is commutative:

$$
\begin{array}{cccc}
\lambda_{I} \otimes_{T} \Omega^{*}(K)^{(n)} & \rightarrow \Omega^{*}(K)^{r(I)} & \stackrel{\alpha_{I}}{\longrightarrow} \Omega^{*}\left(K^{r(I)}\right) & \rightarrow \Omega^{*}\left(D_{I}\right) \\
\downarrow \lambda_{i j} & \downarrow \mu_{i j} & \downarrow \tilde{\mu}_{i j}^{*} & \downarrow \rho^{*} \\
\lambda_{J} \otimes_{T} \Omega^{*}(K)^{(n)} & \rightarrow \Omega^{*}(K)^{r(J)} & \stackrel{\alpha_{J}}{\longrightarrow} \Omega^{*}\left(K^{r(J)}\right) & \rightarrow \Omega^{*}\left(D_{J}\right)
\end{array}
$$

The left, horizontal maps are the inverses of the isomorphism in 2.5. Define $\alpha_{I}$ as follows:

$$
\alpha_{I}\left(x_{1} \otimes \cdots \otimes x_{s}\right)=\pi_{1}^{\#} x_{1} \cup \cdots \cup \pi_{s}^{*} x_{s},
$$

and similarly for $\alpha_{J}$. Then because we are taking commutative cochains, $\alpha_{I}$ and $\alpha_{J}$ are maps of algebras. The map $\tilde{\mu}_{i j}^{\#}$ is induced by the diagonal mapping, while $\mu_{i j}$ is the multiplication. Thus the middle square commutes because of the naturality of the cup product

$$
\begin{aligned}
& \underset{\substack { x_{1} \\
\begin{subarray}{c}{\downarrow \mu_{i j} \\
\mu^{2}{ x _ { 1 } \\
\begin{subarray} { c } { \downarrow \mu _ { i j } \\
\mu ^ { 2 } } }\end{subarray}}{\longrightarrow} \pi_{1}^{*} x_{1} \cup \cdots \cup \pi_{s}^{*} x_{s} \\
& \pm x_{1} \otimes \cdots \otimes x_{i_{0}} x_{j_{0}} \otimes \cdots \otimes \hat{x}_{j_{0}} \otimes \cdots \otimes x_{s} \rightarrow \pm \pi_{1}^{*} x_{1} \cup \cdots \cup \pi_{i_{0}}^{*}\left(x_{i_{0}} x_{j_{0}}\right) \cup \cdots \cup \pi_{s}^{*} x_{s}
\end{aligned}
$$

and the right square commutes because of the homeomorphisms $X^{r(1)} \leftrightarrow D_{I}$ and $X^{r(J)} \leftrightarrow D_{J}$, which are compatible with cup products. Here the signs come about from the graded multiplication. 
Examples of formal spaces are some homogeneous spaces, $k$-connected $4 k$ manifolds, Kähler manifolds, and $k$-connected $3 k$-dimensional spaces. We conjecture that Theorem 3.2 is valid for any field, if $K$ is a $k$-connected $3 k$ dimensional space.

\section{THE SPECTRAL SEQUeNCE OF A COSIMPLICIAL SPACE}

This section is an elaboration of $\S \S 5$ and 7 of Bott-Segal [3]. Recall [4, $\S 9]$ that a cosimplicial space $X=\left\{X_{p}\right\}$ is a family of spaces $X_{p}$ and maps $d^{i}: X_{p} \rightarrow X_{p+1}, \quad i=0, \ldots, p+1, \quad s^{j}: X_{p} \rightarrow X_{p-1}, \quad i=0, \ldots, p-1$, satisfying the relations:

$$
\begin{aligned}
& d^{j} d^{i}=d^{i} d^{j-1}, \quad i<j, \\
& s^{j} s^{i}=s^{i-1} s^{j}, \quad i>j, \\
& s^{j} d^{i}= \begin{cases}d^{i} s^{j-1}, & i<j, \\
\mathrm{id}, & i=j, j+1, \\
d^{i-1} s^{j}, & i>j+1 .\end{cases}
\end{aligned}
$$

The basic example of a cosimplicial space is $\Delta=\left\{\Delta^{n}\right\}$, where $\eta^{i}: \Delta^{n} \rightarrow \Delta^{n+1}$ are the coface and $\varepsilon^{j}: \Delta^{n} \rightarrow \Delta^{n-1}$ are the codegeneracy maps.

A map $f: \mathbf{X} \rightarrow \mathbf{Y}$ of cosimplicial spaces is a sequence of maps $f_{p}: X_{p} \rightarrow Y_{p}$ which commute with the coface and codegeneracy maps $d^{i}$ and $s^{j}$.

The geometric realization of $\mathbf{X}$, denoted by $\operatorname{Tot} \mathbf{X}$ is the space of maps $\operatorname{Hom}(\Delta, \mathbf{X})$ with the induced topology as a subset of $\prod X_{p}^{\Delta_{p}}$.

Example 4.1. Suppose $X \stackrel{f}{\rightarrow} Y \stackrel{g}{\longleftarrow} Z$ is a diagram of spaces. We construct the cosimplicial space of Rector [10], $X \times_{Y} Z$, as follows:

$$
\left(X \times{ }_{Y} Z\right)_{p}=X \times Y^{p} \times Z
$$

with cofaces $d^{i}:\left(X \times_{Y} Z\right)_{p} \rightarrow\left(X \times_{Y} Z\right)_{p+1}$ given by

$$
d^{i}\left(x, y_{1}, \ldots, y_{p}, z\right)= \begin{cases}\left(x, f(x), y_{1}, \ldots, y_{p}, z\right) & \text { if } i=0, \\ \left(x, y_{1}, \ldots, y_{i}, y_{i+1}, \ldots, y_{p}, z\right) & \text { if } 1 \leq i \leq p, \\ \left(x, y_{1}, \ldots, y_{p}, g(z), z\right) & \text { if } i=p+1\end{cases}
$$

The realization, $\operatorname{Tot}\left(X \times_{Y} Z\right)$, is of the same homotopy type as the homotopy pullback $W$

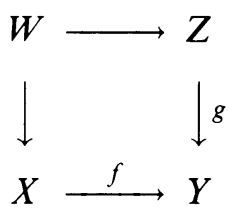

where $W \subset X \times Y^{I} \times Z$ consists of triples $(x, \alpha, z)$ with $\alpha: I \rightarrow Y$ and $\alpha(0)=f(x), \alpha(1)=g(z)$. When $g$ is a fibration, $W$ is of the same homotopy type as the pullback $W^{\prime} \subset X \times Z$ consisting of pairs $(x, z)$ with $f(x)=g(z)$. 
There is a canonical map $W^{\prime} \rightarrow \operatorname{Tot}\left(X \times_{Y} Z\right)$ which is a homotopy equivalence when $g$ is a fibration.

Example 4.2. Suppose that $A$ is a partially ordered set and $X_{\alpha}, \alpha \in A$, is a family of topological spaces. Then the $\left\{X_{\alpha}\right\}$ is called an inverse system of spaces [3, 4], if for every $\alpha \leq \beta$, there exists a map $\phi_{\alpha}^{\beta}: X_{\beta} \rightarrow X_{\alpha}$ with $\alpha \leq \beta \leq \gamma$ implying $\phi_{\alpha}^{\beta} \circ \phi_{\beta}^{\gamma}=\phi_{\alpha}^{\gamma}$. One can form a cosimplicial space $\mathbf{Z}$ as follows:

$$
Z_{p}=\prod_{\alpha_{0} \leq \alpha_{1} \leq \cdots \leq \alpha_{p}} X_{\alpha_{0}}
$$

with coface and codegeneracies induced by the $\phi_{\beta}^{\alpha}$. The space Tot $\mathbf{Z}$ is called the homotopy limit of the system. If $A=A_{1} \cup A_{2}$ and $A_{0}=A_{1} \cap A_{2}$, we have the following induced diagram of fibrations:

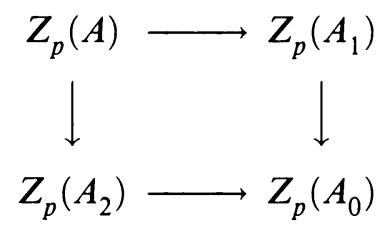

Example 4.3. Suppose $K$ is a simplicial set and $Y$ a topological space. Then the function space $Y^{|K|}$ can be obtained as Tot $Y^{K}$, where $Y^{K}$ is the following cosimplicial space:

$$
\left(Y^{K}\right)_{p}=Y^{K_{p}}=\prod_{\sigma \in K_{p}} Y .
$$

We may think of $Y^{K_{p}}$ as the space of functions from the set $K_{p}$ to $Y$. Then $d^{i}: Y^{K_{p}} \rightarrow Y^{K_{p+1}}$ comes from $K_{p+1} \stackrel{d_{i}}{\rightarrow} K_{p} \rightarrow Y$, and $s^{j}: Y^{K_{p}} \rightarrow Y^{K_{p-1}}$ comes from

$$
K_{p-1} \stackrel{s_{j}}{\longrightarrow} K_{p} \rightarrow Y \text {. }
$$

Proposition 4.4 [3]. There exists a homeomorphism $Y^{|K|} \approx \operatorname{Tot} Y^{K}$.

Given a cosimplicial space $\mathbf{X}$ and a cochain algebra functor $C^{*}, C^{*}(\mathbf{X})$ is a simplicial cochain algebra, i.e., we have cochain maps

$$
C^{*}\left(X_{p}\right) \underset{s^{\prime}}{\stackrel{d^{i}}{\leftrightarrows}} C^{*}\left(X_{p-1}\right)
$$

and we may form a cochain complex $\operatorname{Tot} C^{*}(\mathbf{X})$ [3] as follows: Let

$$
\bar{C}^{*}\left(X_{p}\right)=C^{*}\left(X_{p}\right) /\left(\tilde{s}^{0} C^{*}\left(X_{p-1}\right)+\cdots+\tilde{s}^{p-1} C^{*}\left(X_{p-1}\right)\right)
$$

and

$$
\operatorname{Tot}\left(C^{*}(\mathbf{X})\right)^{n}=\prod_{q-p=n} \bar{C}^{q}\left(X_{p}\right)
$$

with differential

$$
d=\sum(-1)^{i} \tilde{d}^{i}+(-1)^{q+1} \delta .
$$


If now $C^{*}$ is a cochain functor, as in $\S 1$, we will define a natural cochain map

$$
\phi: \operatorname{Tot} C^{*}(\mathbf{X}) \rightarrow C^{*} \operatorname{Tot} \mathbf{X} .
$$

In $\S 5$ we will show that under certain conditions $\phi$ is an equivalence.

We have an evaluation map $e: \Delta^{p} \times \operatorname{Tot} \mathbf{X} \rightarrow X_{p}$ which is a map of cosimplicial spaces. $C^{*}$ has a functorial Eilenberg-Zilber map

$$
C^{*}(X \times Y) \stackrel{\theta}{\rightarrow} C^{*}(X) \otimes C^{*}(Y)
$$

which is a cochain equivalence. Then we form

$$
C^{p+q}\left(X_{p}\right) \stackrel{e^{*}}{\rightarrow} C^{p+q}\left(\Delta^{p} \times \operatorname{Tot} \mathbf{X}\right) \stackrel{\theta_{p}}{\rightarrow}\left[C^{*}\left(\Delta^{p}\right) \otimes C^{*} \operatorname{Tot} \mathbf{X}\right]^{p+q} \stackrel{\phi_{p}}{\rightarrow} C^{q} \operatorname{Tot} \mathbf{X},
$$

where $\phi_{p}$ is the slant product [11] with the canonical $p$-simplex $i_{p} \in C_{p}\left(\Delta^{p}\right)$ and $\phi_{p}(a \otimes b)=a\left(i_{p}\right) b$. Define $\phi=\phi_{p} \circ \theta_{p} \circ e^{\#}$. Thus, if $x \in C^{p+q}\left(X_{p}\right)$, $\phi(x)=e^{\#} x / i_{p}$. From [11, p. 287],

$$
\delta\left(e^{\#} x / i_{p}\right)=\left(\delta e^{\#} x\right) / i_{p}+(-1)^{q} e^{\#} x / \partial i_{p} .
$$

But

$$
e^{\#} \tilde{d} x / i_{p-1}=\sum(-1) e^{\#} \tilde{d}^{i} x / i_{p-1}=\sum(-1)^{i} e^{\#} x / d_{i_{*}} i_{p-1}=e^{\#} x / \partial i_{p}
$$

and $\delta \phi=\phi d$. Because $e$ is a map of cosimplicial spaces, $\phi$ passes to normalized cochains.

We say the cosimplicial space $\mathbf{X}$ is convergent if $\phi$ is a cochain equivalence.

We recall the following consequence of the Eilenberg-Zilber theorem [8, $\mathrm{p}$. 239].

Proposition 4.6. If $X$ and $Y$ are cosimplicial spaces and we have a field for coefficients, then the diagonal cosimplicial space $\left\{X_{p} \times Y_{p}\right\}=D(\mathbf{X}, \mathbf{Y})$ is convergent if both $X$ and $Y$ are convergent.

The following is proven in [3].

Theorem 4.7. Suppose we have pullback diagrams

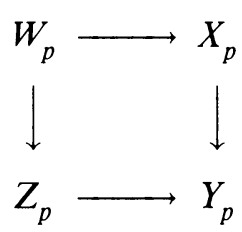

where $X_{p} \rightarrow Y_{p}$ is a fibration. Then $\mathbf{X}=\left\{X_{p}\right\}, \mathbf{Y}=\left\{Y_{p}\right\}, \mathbf{Z}=\left\{Z_{p}\right\}$, and $\mathbf{W}=\left\{W_{p}\right\}$ are cosimplicial spaces and the maps of the squares form maps of cosimplicial spaces. If $Y_{p}$ and $\operatorname{Tot} \mathbf{Y}$ are one-connected and $\mathbf{X}, \mathbf{Y}, \mathbf{Z}$ are convergent, then so is $\mathbf{W}$. 
In the next two sections we will apply the double complex of a cosimplicial space to obtain some collapsing theorem of the associated spectral sequences.

\section{FunCtion SPACES}

In Example 4.2 we saw that if $K$ is a simplicial set, then $Y^{K}$ is a cosimplicial space for any space $Y$. Theorem 4.7 on convergence applies to yield (see [3, p. 296])

Theorem 5.1 (Anderson, Bott-Segal). If $\operatorname{dim}|K|<$ connect $Y$, then $Y^{K}$ is convergent.

Proof. We use induction on the number of nondegenerate simplices of $K$. Assume Theorem 5.1 has been proven for $K_{1}$ and $K_{2}$. Let $K=K_{1} \cup K_{2}$, $K_{0}=K_{1} \cap K_{2} . \quad Y^{K_{0}}$ satisfies the connectivity hypothesis of Theorem 4.7 by induction. We have a commutative fiber square

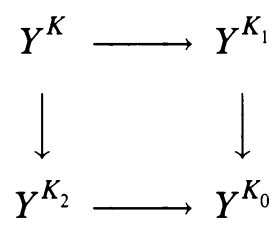

By induction, $Y^{K_{i}}$ is convergent, so Theorem 4.7 gives $Y^{K}$ convergent.

We now construct a functor $F_{K}: \mathscr{D} \rightarrow \mathscr{S} \mathscr{D}$, where $K$ is a simplicial set, $\mathscr{D}$ is the category of commutative DGA-algebras over a field $F$, and $\mathscr{S D}$ is the category of commutative simplicial DGA-algebras. Let $A$ be a DGA-algebra and suppose we give $K_{p}$ a total order for every $p$. Then we form $A^{K_{p}}=$ $A_{\sigma_{1}} \otimes \cdots \otimes A_{\sigma_{n_{p}}}$, where $A_{\sigma_{i}}=A$ and $\sigma_{1}, \ldots, \sigma_{n_{p}}$ run through the $p$-simplices in $K_{p}$ in the chosen order. Define $\partial_{i}: A^{K_{p}} \rightarrow A^{K_{p-1}}$, for $i=0, \ldots, p$, on generators by

$$
\partial_{i}\left(a_{1} \otimes \cdots \otimes a_{n_{p}}\right)=\varepsilon\left(b_{1} \otimes \cdots \otimes b_{n_{p-1}}\right),
$$

where $b_{j}=a_{l_{1}}^{j}, \ldots, a_{l_{r_{j}}}^{j}$ and $\partial_{i}^{-1}\left(\sigma_{j}\right)=\left\{\tau_{l_{1}}^{j}, \ldots, \tau_{l_{r_{j}}}^{j}\right\}$ in the order of $K_{p-1}$. Also $\varepsilon= \pm 1$ and its value is obtained by the sign that is needed to pass from $a_{1} \otimes \cdots \otimes a_{n_{p}}$ to $a_{l_{1}}^{1} \otimes \cdots \otimes a_{l_{r_{1}}}^{1} \otimes a_{l_{1}}^{2} \otimes \cdots \otimes a_{l_{r_{2}}}^{2} \otimes \cdots \otimes a_{l_{1}}^{n_{1}-1} \otimes \cdots \otimes a_{l_{r_{p}}}^{n_{p}-1}$. Note that $\partial_{i}^{-1}\left\{\sigma_{j}\right\}$ is always nonempty as it always contains at least $s_{i} \sigma_{j}$. Also define

$$
A^{K_{p-1}} \stackrel{s_{j}}{\longrightarrow} A^{K_{p}}, \quad j=0,1, \ldots, p-1,
$$

by $s_{j}\left(a_{1} \otimes \cdots \otimes a_{n_{p-1}}\right)=c_{1} \otimes \cdots \otimes c_{n_{p}}$, where

$$
c_{t}= \begin{cases}1 & \text { if } \sigma_{t} \notin \operatorname{Im}\left(s_{j}\right), \\ a_{r} & \text { if } \sigma_{t}=s_{j}\left(\tau_{r}\right) .\end{cases}
$$


It is not hard to verify that $\left\{A^{K_{p}}\right\}$ is a simplicial commutative DGA-algebra, i.e.,

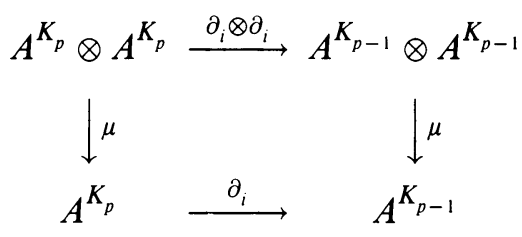

commutes, where

$$
\mu\left(a_{1} \otimes \cdots \otimes a_{n_{p}}\right) \otimes\left(b_{1} \otimes \cdots \otimes b_{n_{p}}\right)=\varepsilon^{\prime} a_{1} b_{1} \otimes \cdots \otimes a_{n_{p}} b_{n_{p}},
$$

where $\varepsilon^{\prime}$ is the usual sign used in the tensor product of algebras, i.e., $(a \otimes b)$. $(c \otimes d)=(-1)^{|b||c|} a c \otimes b d$.

Suppose now that $Y$ is also a simplicial complex and we take $C^{*}(Y)$ to be Sullivan commutative cochains over $Q$. Let us set $A=C^{*}(Y)$. The above process defines a simplicial DGA-algebra $\left\{A^{K_{p}}\right\}$.

Theorem 5.2. There are maps $A^{K_{p}} \rightarrow C^{*}\left(Y^{K_{p}}\right)$ which are equivalences and which induce a map of simplicial cochain algebras.

Proof. Recall that for commutative cochains, the map $C^{*} X \otimes C^{*} Y \stackrel{\phi}{\rightarrow} C^{*}(X \otimes Y)$ given by $\phi(a \otimes b)=\pi_{1}^{\#} a \cup \pi_{2}^{*} b$, where $\pi_{i}$ are the projections, is a natural cochain equivalence $A$-algebra. Define $A^{K_{p}} \stackrel{\phi_{p}}{\rightarrow} C^{*}\left(Y^{K_{p}}\right)$ by $\phi_{p}\left(a_{1} \otimes \cdots \otimes a_{n_{p}}\right)=$ $\pi_{1}^{\#} a_{1} \cup \cdots \cup \pi_{n_{p}}^{\#} a_{n_{p}}$. It is then easy to see that $\phi_{p}$ commutes with faces and degeneracies as they are induced by maps.

From Lemma (5.10) of [3] we see that we may use the double complex $\left\{A^{K_{p}}\right\}$ to compute $H^{*}\left(Y^{|K|}, Q\right)$, when we restrict to the rationals. Immediately, we obtain

Theorem 5.3. If $\operatorname{dim}|K|<\operatorname{connect} Y$, and $Y$ is formal, the spectral sequence for $H^{*}\left(Y^{|K|} ; Q\right)$ collapses at $E_{2}$.

Proof. If the space $Y$ is formal, it means that $A=C^{*}(Y ; Q)$ and $H^{*}(Y ; Q)$ are weakly equivalent, and hence

$$
H^{*}\left(Y^{|K|} ; Q\right) \simeq H^{*}\left(\operatorname{Tot}\left\{H^{*}(Y ; Q)^{K_{p}}\right\}\right) .
$$

\section{THE $E_{1}$-TERM FOR A FUNCTION SPACE}

Recall that if $X$ is a simplicial set, $C_{p}(X)$ is the vector space generated by the simplices of $X_{p}$. The face and degeneracy maps extend to homomorphisms. One may define $N C_{p}(X)=C_{p}(X) \cap \operatorname{Ker} \partial_{1} \cap \cdots \cap \operatorname{Ker} \partial_{p}$; then $N C_{p}(X) \stackrel{\partial_{0}}{\rightarrow}$ $N C_{p-1}(X)$ satisfies $\partial_{0}^{2}=0$ and

$$
H_{*}(N C X) \simeq H_{*}(X) \simeq H_{*}(|X|) .
$$


Also $N^{\prime} C_{p}(X)=N C_{p}(X) /\left(s_{0} C_{p-1}(X)+\cdots+s_{p-1} C_{p-1}(X)\right)$ has induced $\partial^{\prime}$ and again $H_{*}\left(N^{\prime} C X\right) \simeq H_{*}(X)$.

Let $A$ be a commutative DGA-algebra and $K$ a simplicial set of finite type. We now study the double complex $\left\{A^{K_{p}}\right\}$ or its associated double complex $\left\{\overline{A^{K_{p}}}\right\}$ of normalized cochains, i.e.,

$$
\overline{A^{K_{p}}}=A^{K_{p}} /\left(s_{0} A^{K_{p-1}}+\cdots+s_{p-1} A^{K_{p-1}}\right) .
$$

More precisely, $A^{K_{p}}=A_{\sigma_{1}} \otimes \cdots \otimes A_{\sigma_{n_{p}}}$.

Let $\bar{A}_{\sigma} \simeq \bar{A}=\operatorname{Ker}(\varepsilon: A \rightarrow F)$. Then

$$
\overline{A^{K_{p}}}=\bigoplus \otimes\left(\bar{A}_{\sigma_{1}} \otimes \cdots \otimes \bar{A}_{\sigma_{r}}\right),
$$

where the $\sigma_{i} \in K_{p}, \sigma_{i} \notin \operatorname{Im} s_{j}$, and the sum ranges between 0 and $p-1$. But we can also look at

$$
\overline{H^{*}\left(A^{K_{p}}\right)}=H^{*}\left(A^{K_{p}}\right) /\left(s_{0}^{*} H^{*}\left(A^{K_{p-1}}\right)+\cdots+s_{p-1}^{*} H^{*}\left(A^{K_{p-1}}\right)\right)
$$

and by the Kunneth formula we have

$$
\overline{H^{*}\left(A^{K_{p}}\right)} \simeq H^{*} \overline{\left(A^{K_{p}}\right)} .
$$

We have two filtrations on $\left\{\overline{A^{K_{p}}}\right\}$. We look at the spectral sequence coming from $F_{r}=\bigoplus_{p \leq r} \overline{A^{K_{p}}}$. Then

$$
E_{1}^{p}=H^{*}\left(\overline{A^{K_{p}}}\right) \simeq \overline{H^{*}\left(A^{K_{p}}\right)} .
$$

Theorem 6.1. The $E_{1}$-term can be identified as

$$
E_{1}^{p} \simeq \bigoplus_{r=0}^{n_{p}} N^{\prime} C_{p}\left(K^{r}, D^{r} K\right) \otimes_{\Sigma_{r}} H^{*}(A)^{(r)} .
$$

Proof. We look at $H^{*}\left(A^{K_{p}}\right)$. Then relative to the ordering of the simplices of $K_{p}, \sigma_{1}<\cdots<\sigma_{n_{p}}$, we write

$$
\begin{aligned}
H^{*}\left(A^{K_{p}}\right) \simeq & Q \oplus \bigoplus_{\sigma_{i}} \bar{H}^{*}(A) \oplus \bigoplus_{\sigma_{i}<\sigma_{j}} H^{*}(A) \oplus \bar{H}^{*}(A) \\
& \oplus \cdots \oplus \bigoplus_{\sigma_{1}<\sigma_{2}<\cdots<\sigma_{n_{p}}} \bar{H}^{*}(A) \otimes \cdots \otimes \bar{H}^{*}(A) .
\end{aligned}
$$

It is clear that the summand

$$
\bigoplus_{\sigma_{t_{1}}<\cdots<\sigma_{t_{r}}} \bar{H}^{*}(A) \otimes \cdots \otimes \bar{H}^{*}(A)
$$

can be identified with $C_{p}\left(K^{r}, D^{r} K\right) \otimes_{\Sigma_{r}} \bar{H}^{*}(A)^{(r)}$ as $C_{p}\left(K^{r}, D^{r} K\right)$ consists of $\tau_{1} \times \cdots \times \tau_{r}$, where $\tau_{i}$ are $p$-simplices of $K$ and all are distinct. It suffices to see that

$$
\overline{H^{*}\left(A^{K_{p}}\right)} \simeq \bigoplus_{r} N^{\prime} C_{p}\left(K^{r}, D^{r} K\right) \otimes_{\Sigma_{r}} \bar{H}^{*}(A)^{(r)} .
$$


Let $D=s_{0}^{*} H^{*}\left(A^{K_{p-1}}\right)+\cdots+s_{p-1}^{*} H^{*}\left(A^{K_{p-1}}\right)$. By the definition of $s_{j}$ if we study $s_{j}^{*} H^{*}\left(A^{K_{p-1}}\right)$ in the decomposition (6.2), then

$$
s_{j}^{*} H^{*}\left(A^{K_{p-1}}\right) \cap\left[\bigoplus_{\sigma_{i_{1}}<\cdots<\sigma_{i_{r}}}\left(\bar{H}^{*}(A) \otimes \cdots \otimes \bar{H}^{*}(A)\right)\right]
$$

are those summands consisting precisely of $s_{j} \tau_{1}<\cdots<s_{j} \tau_{r}$. Under the identification we have made, it corresponds to simplices $\left(s_{j} \tau_{1}, \ldots, s_{j} \tau_{r}\right)$ with coefficients in $\bar{H}^{*}(A)^{(r)}$, i.e., $D$ goes to zero in $\bigoplus N^{\prime} C_{p}\left(K^{r}, D^{r} K\right) \otimes_{\Sigma_{r}} \bar{H}^{*}(A)^{(r)}$, i.e., we have shown the isomorphism

$$
H^{*}\left(A^{K_{p}}\right) \rightarrow \bigoplus C_{p}\left(K^{r}, D^{r} K\right) \otimes_{\Sigma_{r}} \bar{H}^{*}(A)^{(r)}
$$

passes to give an epimorphism

$$
\overline{H^{*}\left(A^{K_{p}}\right)} \stackrel{\phi}{\rightarrow} \bigoplus N^{\prime} C_{p}\left(K^{r}, D^{r} K\right) \otimes_{\Sigma_{r}} \bar{H}^{*}(Y)^{(r)} .
$$

Similarly one constructs an inverse map $\psi$ to $\phi$ and this establishes the isomorphism.

For the function space $Y^{|K|}$, we have a spectral sequence with

$$
E_{1}^{p} \simeq H^{*}\left(\overline{C^{*}\left(Y^{K_{p}}\right)}\right)
$$

If we show that $H^{*}\left(\overline{C^{*}\left(Y^{K_{p}}\right)} \simeq \overline{H^{*}\left(C^{*}\left(Y^{K_{p}}\right)\right)}\right.$, then the above arguments apply and we obtain

Theorem 6.3. If $\operatorname{dim}|K|<$ connect $Y$ and we take coefficients in a field $F$, there exists a spectral sequence converging to $H^{*}\left(Y^{|K|} ; F\right)$ and with

$$
E_{1}^{p}=\bigoplus_{r=0}^{n p} N^{\prime} C_{p}\left(K^{r}, D K^{r}\right) \otimes_{\Sigma_{r}} \bar{H}^{*}(Y ; F)^{(r)},
$$

where $n_{p}=$ number of $p$-simplices in $K_{p}$.

Proof. The degeneracies in $Y^{K_{p}}$ correspond to projection maps and thus

$$
H^{*}\left(\overline{\left.C^{*}\left(Y^{K_{p}}\right)\right)} \simeq \overline{H^{*}\left(C^{*}\left(Y^{K_{p}}\right)\right)}\right.
$$

follows by Kunneth.

\section{Applications}

In this section we collect some results that follow when we apply the previous theorem to special cases, notably when we take coefficients in the rationals and assume formality. We begin with the more general results. 
Theorem 7.1. If $\operatorname{dim}|K|<\operatorname{connect} Y$ and we take coefficients in a field $F$, we have

(1) A spectral sequence abutting to $H^{*}\left(Y^{|K|} ; F\right)$ with

$$
E_{1}^{p}=\bigoplus_{r=0}^{n p} N^{\prime} C_{p}\left(K^{r}, D^{r} K\right) \otimes_{\Sigma_{r}} \bar{H}^{*}(Y)^{(r)} .
$$

(2) If $Y$ has a trivial cohomology ring, then

$$
E_{2}^{p}=\bigoplus_{r=0}^{n p} H_{p}^{\Sigma_{r}}\left(K^{r}, D^{r} K ; \bar{H}^{*}(Y)^{(r)}\right) .
$$

(3) In general there exists an algebraic spectral sequence $\widetilde{E}_{r}$, whose $\widetilde{E}_{2}$-term is as in (2) and abutting $E_{2}$.

Corollary 7.2. If $F=Q$, then

$$
\widetilde{E}_{2}=H_{*}\left(K^{r}, D^{r} K\right) \otimes_{\Sigma_{r}} \bar{H}^{*}(Y)^{(r)} .
$$

Corollary 7.3. If $F=Q$ and $Y$ is formal, then $E_{2}=E_{\infty}$.

Corollary 7.4. If $F=Q$ and $Y$ is a suspension, then as vector spaces

$$
H^{*}\left(Y^{|K|}\right) \simeq \bigoplus_{q-p=n} H_{p}\left(K^{r}, D^{r} K\right) \otimes_{\Sigma_{r}}\left[H(Y)^{(r)}\right]^{q} .
$$

Corollary 7.5. If $F=Q$ and $|K|$ is formal, then

$$
E_{2}=H_{*}\left(\Lambda\left(r, H^{*}(K)\right) \otimes_{\Sigma_{r}} \bar{H}^{*}(Y)^{(r)} .\right.
$$

Corollary 7.6. If $F=Q$ and $|K|$ is formal and $Y$ is a suspension, then as vector spaces

$$
H^{n}\left(Y^{|K|} ; Q\right)=\bigoplus_{q-p=n} H_{p}\left(\Lambda\left(r, H^{*}(K)\right) \otimes_{\Sigma_{r}}\left[\bar{H}^{*}(Y)^{(r)}\right]^{q} .\right.
$$

The only thing to describe is the algebraic spectral sequence $\widetilde{E}$. This comes about by filtering $E_{1}$ as follows:

$$
F_{k} E_{1}=\bigoplus_{r \leq k} N^{\prime} C_{*}\left(K^{r}, D^{r} K\right) \otimes_{\Sigma_{r}} \bar{H}^{*}(Y)^{(r)} .
$$

We finish this section by showing that we have done things with sufficient generality so that we may include the space of sections of a fibration as BottSegal did (see [3]). Then the two spectral sequences $E_{r}$ and $\widetilde{E}_{r}$ correspond to the ones described by Gelfand-Fuks [6] for the special case of the cohomology of the Lie algebra of vector fields on $M$, which by the Bott-Fuks conjecture is the cohomology of a fiber bundle associated to the tangent bundle of $M$.

We recall from Bott-Segal [3] that if $E \rightarrow X$ is a Hurewicz fibration, and let $\left\{U_{\alpha}\right\}_{\alpha \in A}$ be a covering of $X$, then we may construct a cosimplicial space as follows. Consider the indexing set $A$. Let $\sigma=\left(\alpha_{0}, \ldots, \alpha_{j}\right)$ be a sequence. We define a partial order on the $\alpha$ 's by $\alpha \leq \tau$ if $\operatorname{set}(\sigma) \supseteq \operatorname{set}(\tau)$. Note that if 
we associate $U_{\sigma}$ to $\sigma$, where $U_{\sigma}=U_{\alpha_{0}} \cap \cdots \cap U_{\alpha_{j}}$, then $\sigma \leq \tau$ gives $U_{\sigma} \subset U_{\tau}$. Define a simplicial space $X_{\Sigma}$, where

$$
\left(X_{\Sigma}\right)_{p}=\coprod_{\sigma_{0} \leq \cdots \leq \sigma_{p}} U_{\sigma_{0}} .
$$

Then $\left|X_{\Sigma}\right|$ and $X$ are of the same homotopy type for $X$ a CW-complex.

Let $\Gamma_{\sigma}$ be the space of sections of $E \mid U_{\sigma}$. Then the set $\left\{\Gamma_{\sigma}\right\}$ forms an inverse system of spaces to which one associates a cosimplicial space $\Gamma_{\Sigma}$ as in Example 4.3. Now Tot $\Gamma_{\Sigma} \simeq$ space of sections of $\pi^{*} E$ and if $X$ is a CW-complex, then Tot $\Gamma_{\Sigma} \simeq \Gamma(E)$. By choosing the covering to be contractible, the spaces $\Gamma_{\sigma}$ are all homotopy equivalent to the fiber $F$ of the fibration. Hence if we assume that $\operatorname{dim} X<\operatorname{connect} F$, we can apply (4.7) to see that $\operatorname{Tot} C^{*}\left(\Gamma_{\Sigma}\right)$ converges and can be used to compute $C^{*}(\Gamma(E))$.

However, Bott-Segal defined a smaller cosimplicial space than $\Gamma_{\Sigma}$ to compute $H^{*}(\Gamma(E))$. For any $\sigma \in \Sigma$, let $U^{\sigma}=\bigcup_{\alpha \in \sigma} U_{\alpha}$, where $U^{\sigma}$ is the star of $\sigma$. Then $U^{\sigma}$ is contractible if the covering is contractible. Let $E^{\sigma}=\pi^{-1}\left(U^{\sigma}\right)$; then $\left\{E^{\sigma}\right\}$ forms an inverse system of spaces and [3, (5.7)] shows the associated cosimplicial space $E^{\Sigma}$ is homotopy equivalent to $\Gamma_{\Sigma}$.

If we now do the same analysis as done in $\S \S 5$ and 6 for the spectral sequences of $E^{\Sigma}$ and assume that $Y \rightarrow E \rightarrow X$ is a Hurewicz fibration over a CW-complex $X, \operatorname{dim} X<\operatorname{connect} F$, then Theorem 7.1 generalizes to

Theorem 7.7. There exists a spectral sequence abutting $H^{*}(\Gamma(E) ; F)$ with

$$
E_{1}^{p}=\bigoplus N^{\prime} C_{p}\left(X^{r}, D^{r} X\right) \otimes_{\Sigma_{r}} \bar{H}^{*}(Y)^{(r)} \text {. }
$$

If $Y$ has trivial cohomology, then $E_{2}^{p}$ is the homology of $E_{1}^{p}$ using the boundary operator of $\left(X^{r}, D^{r} X\right)$. In general there exists an algebraic spectral sequence $\widetilde{E}_{r}$ converging to $E_{2}$, whose $\widetilde{E}_{2}$ is $E_{2}$ when we assume $H^{*}(Y ; F)$ has trivial cohomology ring. When $F=Q$ and $Y$ is a suspension, then

$$
E_{2}^{p}=\bigoplus H_{p}\left(X^{r}, D^{r} X\right) \otimes_{\Sigma_{r}} \bar{H}^{*}(Y)^{(r)}
$$

and the higher differentials depend only on the "Pontryagin classes of $E$ ".

The last statement means that if $Y \rightarrow E \rightarrow X$ is induced by a map $X \stackrel{f}{\rightarrow} B G$ for some Lie group $G$, we may look at char $E=f^{*} H^{*}(B G ; Q)$ and the differentials involve classes in char $E$. We will deal with this more precisely in another paper.

\section{BIBLIOGRAPHY}

1. D. W. Anderson, A generalization of the Eilenberg-Moore spectral sequence, Bull. Amer. Math. Soc. 78 (1972), 784-788.

2. K. F. Bödigheimer, Stable splittings of mapping spaces, Math. Gött. Schrift. Sonderforsch. Germ. Anal., 1984.

3. R. Bott and G. Segal, The cohomology of the vector fields on a manifold, Topology 16 (1977), 285-298. 
4. A. K. Bousfield and D. M. Kan, Homotopy limits, completion and localization, Lecture Notes in Math., vol. 304, Springer-Verlag, 1972.

5. F. Cohen and L. Taylor, Computations of Gelfand-Fuks cohomology, the cohomology of function spaces, and the cohomology of configuration spaces, Lecture Notes in Math., vol. 657, Springer-Verlag, 1978, pp. 106-143.

6. I. M. Gelfand and D. B. Fuks, The cohomology of the Lie algebra of tangent vector fields on a smooth manifold. I, II, Functional Anal. Appl. 3 (1969), 32-52; 4 (1970), 23-32.

7. A. Haëfliger, Sur la cohomologie de Gelfand-Fuks, Ann. Sci. Ecole Norm. Sup. (4) 9 (1976), 503-532.

8. S. Mac Lane, Homology, Grundlehren Math. Wiss., vol. 114, Springer-Verlag, 1963.

9. D. McDuff, Configuration spaces of positive and negative particles, Topology 14 (1975), 91-107.

10. D. Rector, Steenrod operations in the Eilenberg-Moore spectral sequence, Comment. Math. Helv. 45 (1970), 540-552.

11. E. Spanier, Algebraic topology, McGraw-Hill, 1966.

12. D. Sullivan, Differential forms and the topology of manifolds, Proc. Internat. Conf. on Topology \& Related Topics, Univ. of Tokyo, 1973.

13. R. Thom, L'homologie des espaces fonctionelles, Colloq. de Topologie Algébrique, Louvain, 1956, pp. 29-39.

Department of Mathematics, Hunter College, CUNY, New York, New York 10021

Department of Mathematics, University of Rochester, Rochester, New York 14627 